\title{
Graph Markov Network for Traffic Forecasting with Missing Data
}

\author{
Zhiyong $\mathrm{Cui}^{\mathrm{a}}$, Longfei Lin ${ }^{\mathrm{b}}$, Ziyuan $\mathrm{Pu}^{\mathrm{a}}$, Yinhai Wang,* \\ ${ }^{a}$ Department of Civil and Environmental Engineering, University of Washington, Seattle, WA 98195 USA \\ ${ }^{b}$ School of Electronic and Information Engineering, Beihang University, 100191 Beijing, China
}

\begin{abstract}
Traffic forecasting is a classical task for traffic management and it plays an important role in intelligent transportation systems. However, since traffic data are mostly collected by traffic sensors or probe vehicles, sensor failures and the lack of probe vehicles will inevitably result in missing values in the collected raw data for some specific links in the traffic network. Although missing values can be imputed, existing data imputation methods normally need long-term historical traffic state data. As for short-term traffic forecasting, especially under edge computing and online prediction scenarios, traffic forecasting models with the capability of handling missing values are needed. In this study, we consider the traffic network as a graph and define the transition between network-wide traffic states at consecutive time steps as a graph Markov process. In this way, missing traffic states can be inferred step by step and the spatial-temporal relationships among the roadway links can be Incorporated. Based on the graph Markov process, we propose a new neural network architecture for spatialtemporal data forecasting, i.e. the graph Markov network (GMN). By incorporating the spectral graph convolution operation, we also propose a spectral graph Markov network (SGMN). The proposed models are compared with baseline models and tested on three realworld traffic state datasets with various missing rates. Experimental results show that the proposed GMN and SGMN can achieve superior prediction performance in terms of both accuracy and efficiency. Besides, the proposed models' parameters, weights, and predicted results are comprehensively analyzed and visualized.
\end{abstract}

Keywords: Traffic forecasting, neural network, missing values, traffic network, graph Markov process, graph convolution

\section{Introduction}

Traffic forecasting, as a challenging topic for both academia and industry, has been under active research, development, and implementation for more than 40 years [1]. Traffic forecasting plays an important role in transportation management and the general planning

\footnotetext{
*Corresponding author

Email addresses: zhiyongc@uw.edu (Zhiyong Cui), linlongfei9858@buaa.edu.cn (Longfei Lin), ziyuanpu@uw.edu (Ziyuan $\mathrm{Pu}$ ), yinhai@uw.edu (Yinhai Wang)
} 
process. With the exponential increase in the volume of traffic data and the computational capability, traffic forecasting methods have been gradually shifting from classical statistical models to data-driven machine learning-based methods [2]. In recent years, the rise of artificial intelligence (AI), especially deep learning methods, has dramatically stimulated the traffic forecasting research field. By leveraging the spatial-temporal patterns contained in immense data resources, many deep neural network models, including recurrent neural network (RNN), convolutional neural network (CNN), generative adversarial network (GAN), etc., have been widely applied in traffic forecasting studies and achieved state-of-the-art prediction performance.

However, since network-wide traffic state data are mostly collected by traffic sensors or probe vehicles, sensor failures or irregular sampling from probe vehicles will result in missing values in the collected data. The missing value issue usually leads to an apparent decline in the forecasting performance, as most of the existing methods for traffic forecasting are not capable of dealing with missing values. Thus, forecasting performance of the models that only accept valid data as input will be significantly affected and limited.

The regular solution for the missing data issue is to conduct data imputation, which targets to estimate the corrupted or missing traffic data. Due to the complex spatial-temporal patterns of traffic data, existing novel and effective data imputation methods, such as the Bayesian tensor decomposition approach [3] and the generative adversarial imputation network [4], usually need large datasets covering a long period of time to achieve good imputation performance. However, a large dataset covering a long period of time is not always available. Further, in the connected vehicle environments or under the edge computing scenarios, online forecasting computations need to be completed in devices with limited storage and computational capabilities. Thus, those solutions requiring large datasets is not feasible for real-time traffic forecasting tasks.

To solve the missing value issue and fulfill traffic forecasting at the same time, traffic forecasting models with the capability of dealing with missing values have also been proposed. However, most of the existing models [5, 6], take the spatial-temporal traffic data as multivariate time series, and thus, they neglect the important spatial influence between the road links in the traffic network. There are several deep learning-based methods [7, 8] taking spatial factors into consideration. However, they still cannot incorporate the intrinsic structure of the traffic network into the traffic forecasting process.

In this study, to overcome the problems mentioned above, we propose a graph Markov network (GMN), which is a new neural network architecture for spatial-temporal data forecasting with missing values. The traffic network is converted into a graph with topological properties. We consider the variations of the traffic states in the traffic network has Markov property and graph localization property. Based on the two properties, the traffic state transition process can be considered as a graph Markov process. The GMN is designed based on the graph Markov process, which inherently incorporates the spatial-temporal relationships among the links in the traffic network. By incorporating the spectral graph convolution operation, we also propose a spectral graph Markov network (SGMN). Experimental results indicate that the proposed SGMN and GMN can achieve superior prediction performance with greater efficiency. 
The contributions of this study can be summarized as follows:

1. We consider the traffic network as a graph and define the transition between networkwide traffic states at consecutive time steps as a graph Markov process.

2. We propose a new neural network structure, i.e. the graph Markov network, based on the proposed graph Markov process for dealing with missing values and forecasting traffic state simultaneously.

3. By incorporating the spectral graph convolution operation, we also propose a spectral graph Markov network.

4. Experiment results tested on three real-world network-wide traffic state datasets show that the proposed models can achieve superior prediction performance in terms of both accuracy and efficiency.

The rest of this paper is organized as follows: the second section describes the related studies on traffic forecasting with missing values. The third section introduces the proposed graph Markov process and the proposed GMN model. The fourth section discusses the experimental results and the concluding remarks are presented in the fifth section.

\section{Literature Review}

Classical traffic forecasting models can generally be classified into two categories, traditional statistical models and computational intelligence, i.e. machine learning-based, models [2]. The statistical methods are mostly parametric approaches, including variants of auto-regressive integrated moving average (ARIMA) models [9], parametric Kalman filtering models [10], and other types of time-series models [11], that are developed based on a predefined model structure with theoretical assumptions and the parameters are calibrated using historical data [12]. With the ability to accommodate the stochastic and non-linear nature of traffic patterns, classical machine learning methods are widely adopted for the traffic forecasting task, such as support vector regression [13], Bayesian network approaches [14]. In recent years, with the rise of AI, the performance of emerging deep learning-based traffic forecasting methods outperform that of classical methods.

\subsection{Deep learning-based traffic forecasting methods}

Since the traffic data contain both spatial and temporal attributes, the deep learningbased methods can be grouped by the ways to deal with spatial-temporal traffic data. One type of studies convert the spatial-temporal data into a 2-dimensional (2D) matrix and use long short-term memory (LSTM) recurrent neural network [15], bi-directional LSTM [16], CNN [17], GAN [18], or a combination of multiple models [19], to extract feature and forecast traffic states. However, a traffic network's spatial features cannot be completely represented by a $2 \mathrm{D}$ matrix. Thus, another type of methods $[20,21]$ is proposed to convert the physical roadway networks as images according to roads' geospatial properties. Although the traffic network images demonstrate the true traffic network structure, those images contain too many noisy pixels and blank pixels without traffic state information. To analyze the traffic network in an efficient way, many studies consider the traffic network as a graph and predict traffic state by incorporating the graph convolutional network [22, 23, 24]. 


\subsection{Deep learning-based traffic forecasting with missing values}

Traffic forecasting performance will be highly influenced by the missing values. A bunch of data imputation methods has been developed to solve the missing values issues, including the probabilistic principal component analysis [25], tensor decomposition-based methods [26, 27, 28], clustering approaches [29, 30]. There are also some deep learning-based data imputation methods proposed in the most recent years, such as denoising stacked autoencoder [7] and generative adversarial imputation network [4]. However, those deep learningbased methods requiring a large dataset covering a long period of time may not be feasible for the short-term traffic forecasting tasks.

To combine the data imputation and traffic forecasting together, a few RNN-based approaches, such as the LSTM-M [8], have been proposed based the GRU-D [31] for processing multivariate time series with missing values. Even though these RNN-based methods can recurrently fill missing values in each time step and forecasting the future traffic state, they cannot capture spatial interactions between road links in the traffic network. Further, the RNN-based methods are uninterpretable like a black box. The models cannot tell the spatial relationship between neighboring links and the links' temporal dependencies between different time steps.

To solve these problems, in this study, we consider the traffic state transition process as a graph Markov process and propose the graph Markov network for the traffic forecasting with missing values. The design of the graph Markov network inherently incorporates the spatial-temporal relationships among the links in the traffic network. The graph Markov network making use of the topological structure of the traffic network can achieve accurate prediction results with efficient training and testing process. The proposed graph Markov process and graph Markov network are introduced in detail in the following section.

\section{Problem Definition and Preliminary}

\subsection{Traffic Forecasting}

A traffic network normally consists of multiple roadway links. The traffic forecasting task targets to predict future traffic states of all (road) links or sensor stations in the traffic network based on historical traffic state data. The collected spatial-temporal traffic state data of a traffic network with $S$ links/sensor-stations can be characterized as a $T$-step sequence $\left[x_{1}, x_{2}, \ldots, x_{t}, \ldots, x_{T}\right] \in \mathbb{R}^{T \times S}$, in which $x_{t} \in \mathbb{R}^{S}$ demonstrates the traffic states of all $S$ links at the $t$-th step. The traffic state of the $s$-th link at time $t$ is represented by $x_{t}^{s}$. In this study, the superscript of a traffic state represents the spatial dimension and the subscript denotes the temporal dimension. The short-term traffic forecasting problem can be formulated as, based on $T$-step historical traffic state data, learning a function $F(\cdot)$ to generate the traffic state at next time step as follows:

$$
F\left(\left[x_{1}, x_{2} \ldots, x_{T}\right]\right)=\left[x_{T+1}\right]
$$




\subsection{Graph Representations of Traffic Network}

Since the traffic network is composed of road links and intersections, it is intuitive to consider the traffic network as an undirected graph consisting of vertices and edges. The graph can be denoted as $\mathcal{G}=(\mathcal{V}, \mathcal{E}, \mathcal{A}, \mathcal{D})$ with a set of vertices $\mathcal{V}=\left\{v_{1}, \ldots, v_{D}\right\}$ and a set of edges $\mathcal{E}$ between vertices. $\mathcal{A} \in \mathbb{R}^{S \times S}$ is a symmetric (typically sparse) adjacency matrix with binary elements, where $\mathcal{A}_{i, j}$ denotes the connectedness between nodes $v_{i}$ and $v_{j}$. The existence of an edge is represented through $\mathcal{A}_{i, j}=\mathcal{A}_{j, i}=1$, otherwise $\mathcal{A}_{i, j}=0\left(\mathcal{A}_{i, i}=0\right)$. Based on $\mathcal{A}$, a diagonal graph degree matrix $\mathcal{D} \in \mathbb{R}^{S \times S}$ describing the number of edges attached to each vertex can be obtained by $\mathcal{D}_{i, i}=\sum_{j=1}^{S} \mathcal{A}_{i, j}$.

The $\mathcal{A}$ can only indicate the relationship between different vertices. In some cases, the vertices' relationship with themselves also needs to be characterized. Thus, we define the self-connection adjacency matrix $\mathbf{A}=\mathcal{A}+I$, i.e. $\mathbf{A}_{i, i}=1$, which implies each vertex in the graph is self-connected. Here, $I \in \mathbb{R}^{S \times S}$ is an identity matrix.

In addition, the connectedness of the graph vertices can also be encoded by the Laplacian matrix, which is essential for spectral graph analysis. The combinatorial Laplacian matrix is defined as $\mathcal{L}=\mathcal{D}-\mathcal{A}$ and the normalized definition is $\mathcal{L}=I-\mathcal{D}^{-1 / 2} \mathcal{A} \mathcal{D}^{-1 / 2}$. Since $\mathcal{L}$ is a symmetric positive semi-definite matrix, it can be diagonalized as $\mathcal{L}=U \Lambda U^{T}$ by its eigenvector matrix $U$ [32], where $U=\left[u_{0}, u_{1}, \ldots, u_{S-1}\right] \in \mathbb{R}^{S \times S}$ and $\Lambda=\operatorname{diag}\left(\lambda_{0}, \lambda_{1}, \ldots, \lambda_{S-1}\right) \in \mathbb{R}^{S \times S}$ is the corresponding diagonal eigenvalue matrix satisfying $\mathcal{L} u_{i}=\lambda_{i} u_{i}$.

In this study, under the traffic forecasting scenario, the attribute on vertex $v_{s}$ (road link $s$ ) at time $t$ is denoted as $x_{t}^{s}$. Given the graph representation of the traffic network, the Equation 1 can be extended as

$$
F\left(\mathcal{G},\left[x_{1}, x_{2} \ldots, x_{T}\right]\right)=\left[x_{T+1}\right]
$$

\subsection{Missing Values}

Traffic state data can be collected by multi-types of traffic sensors or probe vehicles. When traffic sensors fail or no probe vehicles go through road links, the collected traffic state data may have missing values. We use a sequence of masking vectors $\left[m_{1}, m_{2}, \ldots, m_{T}\right] \in$ $\mathbb{R}^{T \times S}$, where $m_{t} \in \mathbb{R}^{S}$, to indicate the position of the missing values in traffic state sequence $\left[x_{1}, x_{2}, \ldots, x_{T}\right]$. The masking vector can be obtained by

$$
m_{t}^{s}=\left\{\begin{array}{l}
1, \text { if } x_{t}^{s} \text { is observed } \\
0, \text { otherwise }
\end{array}\right.
$$

where $x_{t}^{s}$ is the traffic state of $s$-th link at step $t$.

\subsection{Traffic Forecasting with Missing Values}

Missing values in traffic data can be handled by many existing data imputation methods. Most state-of-the-art data imputation methods, such as the Bayesian tensor decomposition approach [27] and the Generative Adversarial Imputation Nets (GAIN) [4], need long-term historical data to capture complicated traffic patterns and fill missing values. However, in real-time environments, especially under the connected autonomous vehicle (CAV) and 
edge computing scenarios, it may not be possible to conduct data imputation on historical data and forecast future traffic states sequentially, because the volume of traffic state data is huge and the computing capability of devices is limited. In these cases, the traffic forecasting models should be able to handle missing values. Taking the missing values into consideration, we can formulate the traffic forecasting as follows

$$
F\left(\mathcal{G},\left[x_{1}, x_{2} \ldots, x_{T}\right],\left[m_{1}, m_{2} \ldots, m_{T}\right]\right)=\left[x_{T+1}\right]
$$

\section{Proposed Approach}

In this section, we first describe several properties of traffic states. Based on that, we propose a graph Markov process to characterize the variations of traffic states. Then, we introduce the proposed graph Markov Network for traffic forecasting with the capability of dealing with missing values.

\subsection{Properties}

A traffic network is a dynamic system and the states on all links keep varying resulted by the movements of vehicles in the system. Thus, we assume the traffic network's dynamic process satisfies the Markov property that the future state of the traffic network is conditional on the present state.

Markov property: The future state of the traffic network $x_{t+1}$ depends only upon the present state $x_{t}$, not on the sequence of states that preceded it. Taking $X_{1}, X_{2}, \ldots, X_{t+1}$ as random variables with the Markov property and $x_{1}, x_{2}, \ldots, x_{t+1}$ as the observed traffic states. The Markov process can be formulated in a conditional probability form as

$$
\operatorname{Pr}\left(X_{t+1}=x_{t+1} \mid X_{1}=x_{1}, X_{2}=x_{2}, \ldots, X_{t}=x_{t}\right)=\operatorname{Pr}\left(X_{t+1}=x_{t+1} \mid X_{t}=x_{t}\right)
$$

where $\operatorname{Pr}(\cdot)$ demonstrates the probability.

However, the transition matrix is temporal dependent, since at the different time of a day, the traffic state's transition pattern should be different. Based on Equation 5, the transition process of traffic states can be formulated in the vector form as

$$
x_{t+1}=P_{t} x_{t}
$$

where $P_{t} \in \mathbb{R}^{S \times S}$ is the transition matrix and $\left(P_{t}\right)_{i, j}$ measures how much influence $x_{t}^{j}$ has on forming the state $x_{t+1}^{i}$.

The transition process defined in Equation 6 does not take the time interval between $x_{t+1}$ and $x_{t}$ into consideration. We denote the time interval between two consecutive time steps of traffic states by $\Delta t$. If $\Delta t$ is small enough $(\Delta t \rightarrow 0)$, the traffic network's dynamic process can be measured as a continuous process and the difference between consecutive traffic states are close to zero, i.e. $\left|x_{t+\Delta t}-x_{t}\right| \rightarrow 0$. However, a long time interval may result in more variations between the present and future traffic states, leading to a more complicated transition process. Since the traffic state data are normally processed into discrete data and the size of transition matrix $P_{t}$ is fixed, we consider that the longer the $\Delta t$ 
is, the lower capability of measuring the actual transition process $P_{t}$ has. Thus, we multiply a decay parameter $\gamma \in(0,1)$ in Equation 6 to represent the temporal impact on transition process as

$$
x_{t+1}=\gamma P_{t} x_{t}
$$

The transition matrix can measure the contributions made by all roadway links on a specific link, which assumes that the state of a roadway link is influenced by all links in the traffic network. However, since vehicles in the traffic network traverse connected road links one by one and traffic states of connected links are transmitted by those vehicles, the traffic state of a link will only be affected by its neighboring links during a short period of time.

Graph localization property: The traffic state of a specific link $s$ in a traffic network is mostly influenced by localized links, i.e. the link $s$ itself and its neighboring links, during a short period of time. The neighboring links refer to the links in the graph within a specific order of hops with respect to the link $s$. With the help of the graph's topological structure, the localization property in the graph can be measured based the adjacency matrix in two ways: (1) The self-connection adjacency matrix $\mathbf{A}$, describing the connectedness of vertices, can inherently indicate the localization property of all vertices in the graph. Then, the impacts of localized links can be easily measured by a weighted self-connection adjacency matrix. (2) The other way is to conduct the spectral graph convolution operation on the traffic state $x_{t}$ to measure the localized impacts in the graph. The spectral graph convolution on $x_{t}$ can be defined as $U \Lambda_{\theta} U^{T} x_{t}$ [32], where $U$ is the eigenvector matrix of the Laplacian matrix $\mathcal{L}$ and $\Lambda_{\theta}$ is a learnable diagonal weight matrix.

Graph Markov Process: With the aforementioned two properties, we define the traffic state transition process as a graph Markov process (GMP). The graph Markov process can be formulated in a conditional probability form as

$$
\operatorname{Pr}\left(X_{t+1}=x_{t+1}^{u} \mid X_{t}=x_{t}\right)=\operatorname{Pr}\left(X_{t+1}=x_{t+1}^{u} \mid X_{t}=x_{t}^{v}, v \in \mathcal{N}(u)\right)
$$

where the superscripts $u$ and $v$ are the indices of graph links (road links). The $\mathcal{N}(u)$ denotes a set of neighboring links of link $u$ and link $u$ itself. The properties of this graph Markov process is similar to the properties of the Markov random field [33] with temporal information. If we assume that road links are only influenced by their one-hop neighbors in the graph, based on Equation 7, we can easily incorporate the graph localization properties into the traffic states' transition process by element-wise multiplying the transition matrix $P_{t}$ with the self-connection adjacency matrix $\mathbf{A}$. Then, the GMP can be formulated in the vector form as

$$
x_{t+1}=\gamma\left(\mathbf{A} \odot P_{t}\right) x_{t}
$$

where $\odot$ is the Hadamard (element-wise) product operator that $\left(\mathbf{A} \odot P_{t}\right)_{i j}=\mathbf{A}_{i j} \times\left(P_{t}\right)_{i j}$.

The graph localization property can also be incorporated in the transition process by replacing the transition weight matrix $P_{t}$ with the spectral graph convolution operation. Then, we define the spectral version of the graph Markov process (SGMP) as

$$
x_{t+1}=\gamma U \Lambda_{\theta_{t}} U^{T} x_{t}
$$

where $\Lambda_{\theta_{t}} \in \mathbb{R}^{S \times S}$ is a diagonal weight matrix. 

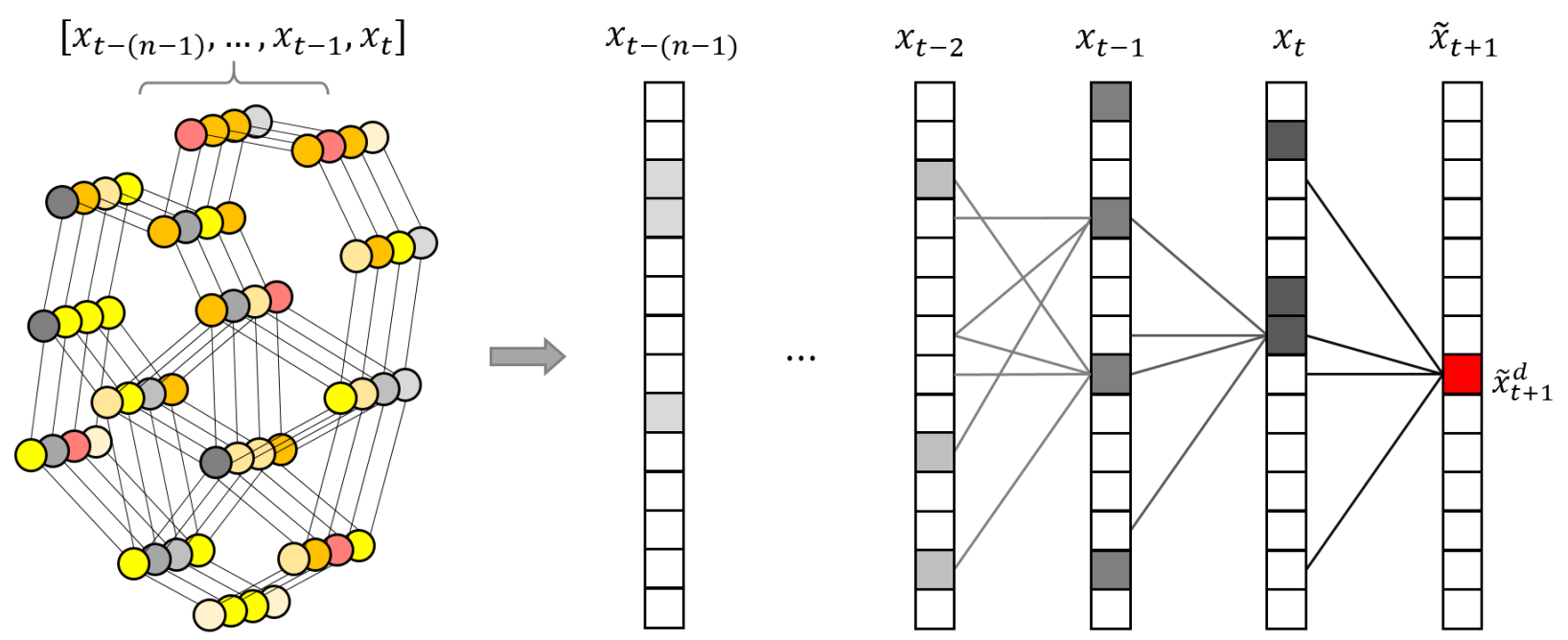

Figure 1: Graph Markov process. The gray-colored nodes in the left sub-figure demonstrating the nodes with missing values. The traffic states are represented by vectors on the right side. A future state (in red color) and the missing states (in gray color) can be inferred from their neighbors in the previous step.

\subsection{Handling Missing Values in the Graph Markov Process}

In this section, we theoretically introduce how to deal with the missing values in the graph Markov process.

As we assume the traffic state transition process follows the graph Markov process, the future traffic state can be inferred by the present state. If there are missing values in the present state, we can infer the missing values from previous states. We consider $x_{t}$ is the observed traffic state at time $t$ and a mask vector $m_{t}$ can be acquired according to Equation 3. We denote the completed state by $\tilde{x}_{t}$, in which all missing values are filled based on historical data. Hence, the completed state consists of two parts, including the observed state values and the inferred state values, as follows:

$$
\tilde{x}_{t}=x_{t} \odot m_{t}+\tilde{x}_{t} \odot\left(1-m_{t}\right)
$$

where $\tilde{x}_{t} \odot\left(1-m_{t}\right)$ is the inferred part. Since $x_{t} \odot m_{t}=x_{t}$, Equation 11 can be written as

$$
\tilde{x}_{t}=x_{t}+\tilde{x}_{t} \odot\left(1-m_{t}\right)
$$

Since the transition of completed states follows the the graph Markov process, the GMP and SGMP with respect to the completed state can be described as $\tilde{x}_{t+1}=\gamma\left(\mathbf{A} \odot P_{t}\right) \tilde{x}_{t}$ and $\tilde{x}_{t+1}=\gamma U \Lambda_{\theta_{t}} U^{T} \tilde{x}_{t}$, respectively. In this section, for simplicity, we denote the (spectral) graph Markov transition matrix by $H_{t}$, i.e. $H_{t}=\mathbf{A} \odot P_{t}$ or $H_{t}=U \Lambda_{\theta_{t}} U^{T}$. Hence, the transition process of completed states can be represented as

$$
\tilde{x}_{t+1}=\gamma H_{t} \tilde{x}_{t}
$$

Applying Equation 12, the transition process becomes

$$
\tilde{x}_{t+1}=\gamma H_{t}\left(x_{t}+\tilde{x}_{t} \odot\left(1-m_{t}\right)\right)
$$


If we iteratively apply the completed state $\tilde{x}_{t}$, i.e. $\tilde{x}_{t}=\gamma H_{t-1}\left(x_{t-1}+\tilde{x}_{t-1} \odot\left(1-m_{t-1}\right)\right)$, into Equation 14 itself, we have

$$
\begin{aligned}
\tilde{x}_{t+1} & =\gamma H_{t}\left(x_{t}+\gamma H_{t-1}\left(x_{t-1}+\tilde{x}_{t-1} \odot\left(1-m_{t-1}\right)\right) \odot\left(1-m_{t}\right)\right) \\
& =\gamma H_{t} x_{t}+\gamma^{2} H_{t} H_{t-1}\left(x_{t-1} \odot\left(1-m_{t}\right)\right)+\gamma^{2} H_{t} H_{t-1}\left(\tilde{x}_{t-1} \odot\left(1-m_{t-1}\right) \odot\left(1-m_{t}\right)\right)
\end{aligned}
$$

After iteratively applying $n$ steps of previous states from $x_{t-(n-1)}$ to $x_{t}, \tilde{x}_{t+1}$ can be described as

$$
\begin{aligned}
\tilde{x}_{t+1} & =\gamma H_{t} x_{t} \\
& +\gamma^{2} H_{t} H_{t-1}\left(x_{t-1} \odot\left(1-m_{t}\right)\right) \\
& +\gamma^{3} H_{t} H_{t-1} H_{t-2}\left(x_{t-2} \odot\left(1-m_{t-1}\right) \odot\left(1-m_{t}\right)\right)+\cdots \\
& +\gamma^{n} H_{t} \cdots H_{t-(n-1)}\left(x_{t-(n-1)} \odot\left(1-m_{t-(n-2)}\right) \odot \cdots \odot\left(1-m_{t}\right)\right) \\
& +\gamma^{n} H_{t} \cdots H_{t-(n-1)}\left(\tilde{x}_{t-(n-1)} \odot\left(1-m_{t-(n-1)}\right) \odot \cdots \odot\left(1-m_{t}\right)\right)
\end{aligned}
$$

The $n$ steps of historical steps of states can be written in a summation form as

$$
\begin{aligned}
\tilde{x}_{t+1} & =\sum_{i=0}^{n-1} \gamma^{i+1}\left(\prod_{j=0}^{i} H_{t-j}\right)\left(x_{t-i} \odot \bigodot_{j=0}^{i-1}\left(1-m_{t-j}\right)\right) \\
& +\gamma^{n} H_{t} \cdots H_{t-(n-1)}\left(\tilde{x}_{t-(n-1)} \odot\left(1-m_{t-(n-1)}\right) \odot \cdots \odot\left(1-m_{t}\right)\right)
\end{aligned}
$$

where $\sum, \prod$, and $\odot$ are the summation, matrix product, and Hadamard product operators, respectively. For simplicity, we denote the term with the $\tilde{x}_{t-n}$ in Equation 17 as $\mathcal{O}\left(\tilde{x}_{t-n}\right)$, and the GMP of the complected states can be represented by

$$
\tilde{x}_{t+1}=\sum_{i=0}^{n-1} \gamma^{i+1}\left(\prod_{j=0}^{i} H_{t-j}\right)\left(x_{t-i} \odot \bigodot_{j=0}^{i-1}\left(1-m_{t-j}\right)\right)+\mathcal{O}\left(\tilde{x}_{t-(n-1)}\right)
$$

In $\mathcal{O}\left(\tilde{x}_{t-(n-1)}\right)$, when $n \rightarrow \infty$, since $\gamma \in(0,1), \gamma^{n+1} \rightarrow 0$. In addition, the product of masking vectors in $\mathcal{O}\left(\tilde{x}_{t-(n-1)}\right)$ will also approach to zero, i.e. $\bigodot_{j=0}^{n-1}\left(1-m_{t-j}\right) \rightarrow 0$. If the missing rate of the traffic state values is $10 \%$ and the missing pattern is random, the probability of each element of $\bigodot_{j=0}^{n-1}\left(1-m_{t-j}\right)$ being zero is $10^{n}$. Thus, when $n$ is large enough, we consider the $\mathcal{O}\left(\tilde{x}_{t-(n-1)}\right)$ is a negligibly term.

Figure 1 demonstrates the graph Markov process for inferring the future state. The traffic network graphs with attribute-missed nodes (in gray color) is convert into traffic state vectors. The inference of $\tilde{x}_{t+1}^{d}$ is based on historical traffic states by back-propagating to the $t-(n-1)$ step.

\subsection{Graph Markov Network}

In this section, we propose a Graph Markov Network (GMN) for traffic prediction with the capability of handling missing values in historical data. Suppose the historical 


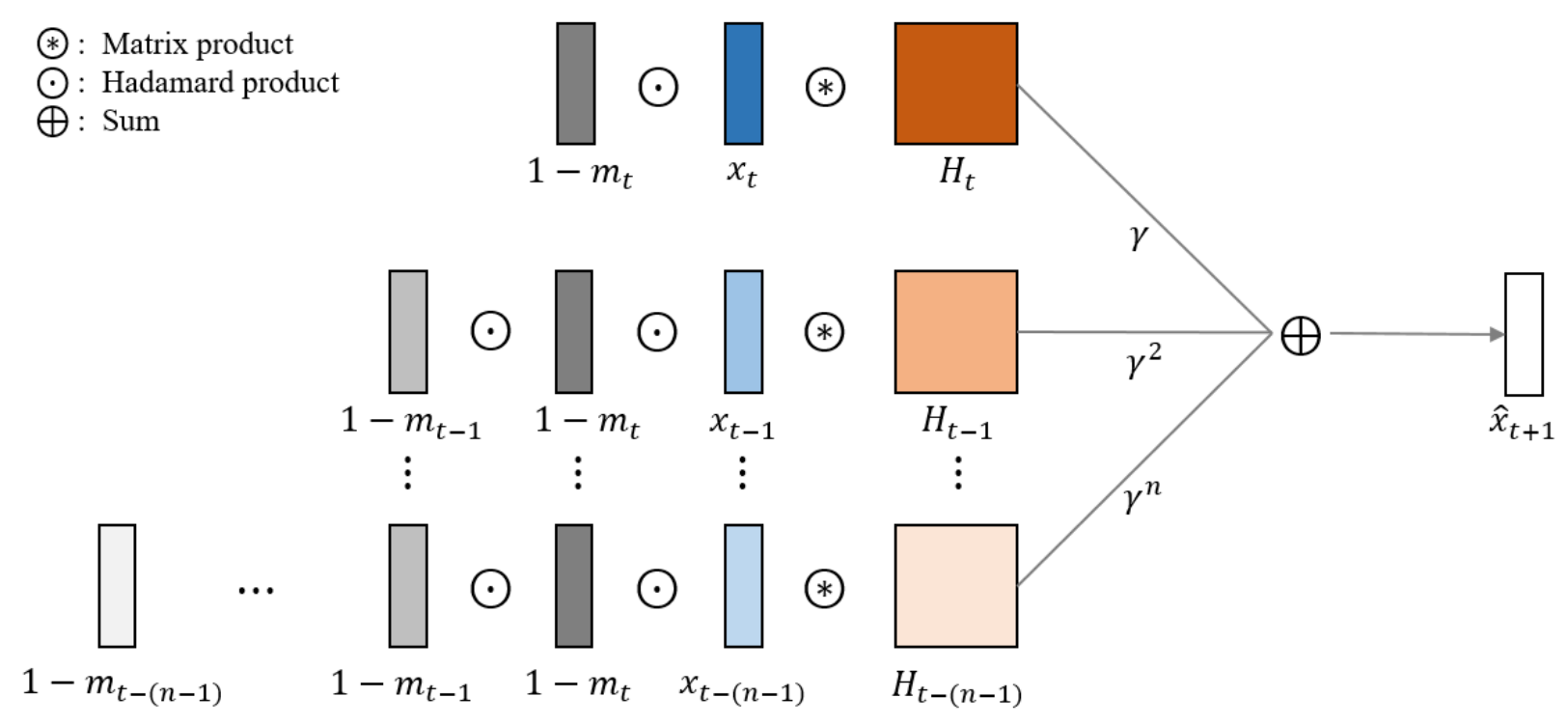

Figure 2: Structure of the proposed graph Markov network. Here, $H_{t-j}=\mathbf{A}^{j} \odot W_{j}$. As for the spectral version of $\mathrm{GMN}, H_{t-j}=U \wedge_{j} U^{T}$.

traffic data consists of $n$ steps of traffic states $\left\{x_{t-(n-1)}, \ldots, x_{t}\right\}$. Correspondingly, we can acquire $n$ masking vectors $\left\{m_{t-(n-1)}, \ldots, m_{t}\right\}$. The traffic network's topological structure can be represented by the adjacency matrix.

The GMN is designed based on the proposed GMP described in the previous section. Since we consider the term $\mathcal{O}\left(\tilde{x}_{t-(n-1)}\right)$ described in Equation 18 is small enough, the $\mathcal{O}\left(\tilde{x}_{t-(n-1)}\right)$ is omitted in the proposed GMN for simplicity.

As described in Equation 18, the graph Markov process contains $n$ transition weight matrices and the product of the these matrices $\left(\prod_{j=0}^{i} H_{t-j}\right)=\left(\prod_{j=0}^{i} \mathbf{A}^{j} \odot P_{t-j}\right)$ measures the contribution of $x_{t-i}$ for generating the $\tilde{x}_{t}$. To reduce matrix product operations and at the same time keep the learning capability in the GMP, we simplify the $\left(\prod_{j=0}^{i} \mathbf{A}^{j} \odot P_{t-j}\right)$ by $\left(\mathbf{A}^{i+1} \odot W_{i+1}\right)$, where $W_{i+1} \in \mathbb{R}^{S \times S}$ is a weight matrix. In this way, $\left(\mathbf{A}^{i+1} \odot W_{i+1}\right)$ can directly measure the contribution of $x_{t-i}$ for generating the $\tilde{x}_{t}$ and skip the intermediate state transition processes. Further, the GMP still has $n$ weight matrices $\left(\left\{\mathbf{A}^{1} \odot W_{1}, \ldots, \mathbf{A}^{n} \odot W_{n}\right\}\right)$, and thus, the learning capability in terms of the size of parameters does not change. The benefits of the simplification is that the GMP can reduce $\frac{n(n-1)}{2}$ times of multiplication between two $S \times S$ matrices in total.

Based on the GMP and the aforementioned simplification, we propose the graph Markov network for traffic forecasting with the capability of handling missing values as

$$
\hat{x}_{t+1}=\sum_{i=0}^{n-1} \gamma^{i+1}\left(\mathbf{A}^{i+1} \odot W_{i+1}\right)\left(x_{t-i} \odot \bigodot_{j=0}^{i-1}\left(1-m_{t-j}\right)\right)
$$

where $\hat{x}_{t+1}$ is the predicted traffic state for the future time step $t+1$ and $\left\{W_{1}, \ldots, W_{n}\right\}$ are the model's weight matrices that can be learned and updated during the training process. 
As for the spectral version of the graph Markov process, the product of the transition weight matrices $\left(\prod_{j=0}^{i} H_{t-j}\right)$ can also be simplified. Because of the orthogonality of the eigenvectors of $\mathcal{L}$ [34], $U^{T}=U^{-1}$, and thus, $\left(\prod_{j=0}^{i} H_{t-j}\right)=\left(\prod_{j=0}^{i} U \Lambda_{\theta_{t-j}} U^{T}\right)=U\left(\prod_{j=0}^{i} \Lambda_{\theta_{t-j}}\right) U^{T}$. We further simplify the product of the transition weight matrices by replacing the $\prod_{j=0}^{i} \Lambda_{\theta_{t-j}}$ with a diagonal weight matrix $\Lambda_{i+1}$. Similar to the simplification of GMP, this simplification process will not reduce the learning capability of the SGMP because the amount of the weight parameters does not change. In this way, the SGMP can reduce at least $\frac{n(n-1)}{2}$ times of vector multiplication in total. Then, the spectral version of the graph Markov network (SGMN) can be defined as

$$
\hat{x}_{t+1}=\sum_{i=0}^{n-1} \gamma^{i+1}\left(U \Lambda_{i+1} U^{T}\right)\left(x_{t-i} \odot \bigodot_{j=0}^{i-1}\left(1-m_{t-j}\right)\right)
$$

where $\left\{\Lambda_{1}, \ldots, \Lambda_{n}\right\}$ are the diagonal weight matrices that can be learned and updated during the training process.

The structure of GMN for predicting traffic state $x_{t+1}$ is demonstrated in Figure 2. The spectral version of GMN has the same model structure that it only need to replace the $\mathbf{A}^{j} \odot W_{j}$ with the $U \wedge_{j} U^{T}$, as shown Figure 2. During the training process, the loss can be calculated by measuring the difference between the predicted value $\hat{y}=\hat{x}_{t+1}$ and the label $y=x_{t+1}$.

\section{Experimental Results}

In this section, we compared the proposed approach with state-of-the-art traffic forecasting models with the capability of handling missing values. The datasets, hyper-parameters, software, and hardware used in the experiments are introduced.

\subsection{Datasets}

In this study, we conduct experiments on three real-world network-wide traffic state datasets. The topological structures of the traffic networks are also used in the experiments.

\subsubsection{PEMS-BAY}

This dataset named as PEMS-BAY is collected by California Transportation Agencies (CalTrans) Performance Measurement System (PeMS). This dataset contains the speed information of 325 sensors in the Bay Area lasting for six months ranging from Jan 1st, 2017 to Jun 30th, 2017. The interval of time steps is 5-minutes. The total number of observed traffic data points is 16,941,600. The adjacency matrix of this dataset is defined according to [35]. The dataset is published by [35] on the Github (https://github.com/ liyaguang/DCRNN). 


\subsection{2. $M E T R-L A$}

This dataset is collected from loop detectors on the freeway of Los Angeles County [36]. This dataset contains the speed information of 207 sensors lasting for 4 months ranging from Mar 1st, 2012 to Jun 30th, 2012. The interval of time steps is 5-minutes. The total number of observed traffic data points is 6,519,002. Similar to the PEMS-BAY dataset, the adjacency matrix of this dataset is defined according to [35], and the dataset is published on the Github (https://github.com/liyaguang/DCRNN).

\subsubsection{INRIX-SEA}

This dataset is collected by the INRIX company from multiple data sources, including GPS probes, road sensors, and cell phone data. This dataset contains the speed information of the traffic network in the Seattle downtown area consisting of 725 road segments. The traffic network covers both freeways and urban roadways. The dataset covers a one-year period from Jan 1st, 2012 to Dec 31st, 2012. The interval of time steps is 5-minutes. The total number of observed traffic data points is $76,212,000$. This dataset is provided by Washington Department of Transportation and has been used in [23]. Due to privacy policies, this dataset is not published.

\subsection{Missing Values and Data formatting}

The dataset forms as a spatial-temporal matrix, whose spatial dimension size is the number of sensors and temporal dimension size is the number of time steps. In the experiments, the dataset is split into a training set, a validation set, and a testing set, with a size ratio of $6: 2: 2$. In the training and testing process, the speed values of the dataset are normalized within a range of $[0,1]$.

The PEMS-BAY and METR-LA datasets originally have missing values and their percentages of missing values are $0.003 \%$ and $8.11 \%$, respectively. There are no missing values in the INRIX-SEA dataset. To test the model's capability of handling missing values with different missing rates, we randomly set a portion of speed values in the input sequences as zeros according to a specific missing rate and generate the masking vectors accordingly. In this study, based on each of the three datasets, three sub-datasets with missing rates of $10 \%, 20 \%$, and $40 \%$, respectively, are generated.

\subsection{Hardware and Software Environments}

The experiments are conducted on a computer with 128GB memory, a Intel Core i97900X CPU, and a NVIDIA GTX 1080 Ti GPU. The proposed approach and all neural network-based baseline models are implemented based on Pytorch 1.0.1 using the Python language 3.6.8.

\subsection{Baseline Models}

- GRU [37]: GRU referring to gated recurrent units is a type of RNN. GRU can be considered as a simplified LSTM. 
- GRU-I : GRU-I is designed based on GRU. Since GRU is a type of a RNN with the recurrent structure, the predicted values from a previous step $\hat{x}_{t}$ can be used to infer the missing values in the next step. The completed traffic states with all missing values filled can be represented by $\tilde{x}_{t+1}=x_{t}+\hat{x}_{t} \odot\left(1-m_{t}\right)$.

- GRU-D [31]: GRU-D is a neural network structure that is designed based on GRU for multivariate time series prediction. It can capture long-term temporal dependencies in time series. GRU-D incorporates the masking information and missing values' time interval as input such that it can utilize the missing patterns.

- LSTM [38]: LSTM is a powerful variant of RNN, which can overcome the gradients exploding or vanishing problem. It is suitable for being a model's basic structure for traffic forecasting.

- LSTM-I : LSTM-I is designed based on LSTM. The missing value inferring process of LSTM-I is similar to that of GRU-I.

- LSTM-M [8]: LSTM-M is a neural network structure designed based on LSTM for traffic prediction with missing data. It employs multi-scale temporal smoothing methods to infer lost data.

\subsection{Model Parameters}

The batch size of the tested data is set as 64 . The number of steps of historical data incorporated in the GMN model will have an influence on the prediction performance. Hence,the GMNs with 6-steps, 8-steps, and 10-steps of historical data are tested in the experiments, i.e. the $n$ in Equations 19 and 20 are set as 6, 8, and 10. In the following sections, we denoted these GMN models as GMN-6, GMN-8, and GMN-10, respectively. The corresponding SGMN models with different steps are denoted as SGMN-6, SGMN-8, and SGMN-10, respectively. The decay parameter $\gamma$ is set as 0.9 in the experiments. For the RNN-based baseline models, including GRU, GRU-I, GRU-D, LSTM, LSTM-I, and LSTM-M, their input sequences all have 10 time steps.

\subsection{Training and Hyper-Parameters}

In the training process, the mean square error (MSE) between the label $y_{t}$ and the predicted value $\hat{y}_{t}$, i.e. $\frac{1}{n} \sum_{i=1}^{N}\left(y_{i}-\hat{y}_{i}\right)^{2}$ is used as the loss function, where $N$ is the sample size. The Adam [39] optimization method is adopted for both GMN models and baseline models to update parameters, as Adam is also used in [31, 8]. Early stopping mechanism is used to avoid over-fitting. If there is no improvement in 5 consecutive epochs, the training will be stopped. The minimum improvement in MSE is set as 0.00001 . We also design a learning rate decay mechanism for the training process to speed up the models' convergence. The initial learning rate of all models is set as $10^{-3}$, which is identical to the learning rate in [8]. If there is no improvement in 4 consecutive epochs, the learning rate is reduced an order of magnitude until it reaches $10^{-5}$. 


\subsection{Evaluation Metric}

The prediction accuracy of all tested models are evaluated by three metrics, including mean absolute error (MAE), mean absolute percentage error (MAPE), and root mean square error (RMSE).

$$
\begin{gathered}
M A E=\frac{1}{N} \sum_{i=1}^{N}\left|y_{i}-\hat{y}_{i}\right| \\
M A P E=\frac{1}{N} \sum_{i=1}^{N}\left|\frac{y_{i}-\hat{y}_{i}}{y_{i}}\right| \\
R M S E=\left(\frac{1}{N} \sum_{i=1}^{N}\left|y_{i}-\hat{y}_{i}\right|^{2}\right)^{\frac{1}{2}}
\end{gathered}
$$

\begin{tabular}{|c|c|c|c|c|c|c|c|c|c|}
\hline \multicolumn{10}{|c|}{ PEMS-BAY } \\
\hline \multirow{2}{*}{ Model } & \multicolumn{3}{|c|}{ Missing Rate $=10 \%$} & \multicolumn{3}{|c|}{ Missing Rate $=20 \%$} & \multicolumn{3}{|c|}{ Missing Rate $=40 \%$} \\
\hline & MAE & MAPE(\%) & RMSE & MAE & MAPE(\%) & RMSE & MAE & MAPE(\%) & RMSE \\
\hline "GRU & 1.608 & 3.133 & 2.608 & 1.787 & 3.522 & 2.911 & 2.052 & 4.095 & 3.320 \\
\hline GRU-I & 1.108 & 133 & 1.831 & .185 & 2.296 & 1.968 & 1.385 & 2.729 & 2.327 \\
\hline GRU-D & 5.320 & 13.584 & 9.163 & 5.347 & 13.609 & 9.160 & 5.387 & 13.67 & 9.180 \\
\hline LSTM & 2.368 & 4.809 & 3.952 & 2.457 & 5.098 & 4.258 & 2.428 & 5.117 & 4.181 \\
\hline LSTM-I & 2.218 & 4.001 & 7.472 & 2.373 & 4.278 & 7.742 & 2.058 & 3.989 & 5.863 \\
\hline LSTM-D & 1.198 & 2.351 & 1.968 & 1.236 & 2.437 & 2.055 & 1.472 & 2.904 & 3.111 \\
\hline GMN-6 & 1.084 & 2.077 & 2.565 & 1.101 & 2.145 & 2.029 & 1.819 & 3.634 & 3.543 \\
\hline GMN-8 & 1.089 & 2.086 & 2.611 & 1.196 & 2.297 & 2.827 & 1.376 & 2.730 & 2.678 \\
\hline GMN-10 & 1.089 & 2.086 & 2.614 & 1.202 & 2.308 & 2.864 & 1.327 & 2.615 & 2.470 \\
\hline SGMN-6 & 1.009 & 930 & 1.877 & 1.064 & 2.048 & 2.067 & 1.930 & 3.671 & 4.375 \\
\hline SGMN-8 & 1.008 & 1.929 & 1.875 & 1.062 & 2.043 & 2.024 & 1.291 & 2.517 & 2.867 \\
\hline SGMN-10 & 1.007 & 1.927 & 1.874 & 1.058 & 2.037 & 2.018 & 1.207 & 2.362 & 2.473 \\
\hline
\end{tabular}

5.8. Experimental Results

Table 1: Prediction performance on PEMS-BAY dataset

\subsubsection{Comparing with baseline models}

The prediction results tested on the PEMS-BAY, METR-LA, and INRIX-SEA datasets with respect to different missing rates are displayed in Table 1, Table 2, and Table 3, respectively. Overall, the SGMN models are superior to other baseline models. The GMN models also perform well, especially on the PEMS-BAY dataset. However, the prediction performance of GMN models decreases faster than that of SGMN models along with the increase of the missing rate. Among the baseline models, the GRU-I model performs well that it achieve smaller RMSEs on the PEMS-BAY and INRIX-SEA datasets.

As shown in Table 1, the SGMN-10 achieves the smallest MAEs and MAPEs for all the three missing rates on the PEMS-BAY dataset. However, the RMSEs of GRU-I are the 
Table 2: Prediction performance on METR-LA dataset

\begin{tabular}{|l||c|c|c|c|c|c|c|c|c|}
\hline \multicolumn{10}{|c|}{ METR-LA } \\
\hline \multirow{2}{*}{ Model } & \multicolumn{1}{|c|}{ Missing Rate $=10 \%$} & \multicolumn{3}{c|}{ Missing Rate $=20 \%$} & \multicolumn{3}{c|}{ Missing Rate $=40 \%$} \\
\cline { 2 - 11 } & MAE & MAPE(\%) & RMSE & MAE & MAPE(\%) & RMSE & MAE & MAPE(\%) & RMSE \\
\hline \hline GRU & 3.427 & 7.971 & 5.923 & 3.667 & 8.611 & 6.249 & 4.037 & 9.622 & 6.744 \\
\hline GRU-I & 3.322 & 7.625 & 5.543 & 3.402 & 7.846 & 5.642 & $\mathbf{3 . 3 8 9}$ & 7.917 & 5.903 \\
\hline GRU-D & 9.912 & 25.28 & 12.195 & 9.904 & 25.302 & 12.193 & 10.022 & 25.444 & 12.269 \\
\hline LSTM & 3.477 & 8.050 & 6.015 & 3.652 & 8.559 & 6.263 & 3.899 & 9.300 & 6.663 \\
\hline LSTM-I & 3.180 & 7.228 & 5.363 & $\mathbf{3 . 2 6 7}$ & 7.417 & 5.653 & 3.393 & $\mathbf{7 . 8 2 6}$ & 5.879 \\
\hline LSTM-D & 3.253 & 7.374 & 5.540 & 3.368 & 7.666 & 5.717 & 3.410 & 7.837 & $\mathbf{5 . 8 1 2}$ \\
\hline GMN-6 & 3.384 & 7.300 & 5.624 & 3.477 & 7.488 & 5.583 & 3.913 & 8.518 & 6.362 \\
\hline GMN-8 & 3.565 & 7.684 & 6.126 & 3.653 & 7.852 & 6.001 & 3.864 & 8.365 & 6.083 \\
\hline GMN-10 & 3.708 & 7.969 & 6.512 & 3.792 & 8.131 & 6.411 & 3.961 & 8.518 & 6.216 \\
\hline SGMN-6 & $\mathbf{3 . 1 4 5}$ & $\mathbf{6 . 8 3 6}$ & 5.331 & 3.333 & 7.232 & 5.578 & 3.952 & 8.593 & 6.894 \\
\hline SGMN-8 & 3.174 & 6.889 & 5.362 & 3.318 & 7.203 & 5.552 & 3.699 & 8.053 & 6.186 \\
\hline SGMN-10 & 3.152 & 6.852 & $\mathbf{5 . 3 2 1}$ & 3.310 & $\mathbf{7 . 1 8 7}$ & $\mathbf{5 . 5 2 5}$ & 3.680 & 8.005 & 6.079 \\
\hline
\end{tabular}

Table 3: Prediction performance on INRIX-SEA dataset

\begin{tabular}{|c|c|c|c|c|c|c|c|c|c|}
\hline \multicolumn{10}{|c|}{ INRIX-SEA } \\
\hline \multirow{2}{*}{ Model } & \multicolumn{3}{|c|}{ Missing Rate $=10 \%$} & \multicolumn{3}{|c|}{ Missing Rate $=20 \%$} & \multicolumn{3}{|c|}{ Missing Rate $=40 \%$} \\
\hline & MAE & MAPE(\%) & RMSE & MAE & MAPE(\%) & RMSE & MAE & MAPE(\%) & RMSE \\
\hline GRU & 1.097 & 3.964 & 2.158 & 1.146 & 4.143 & 2.257 & 1.256 & 4.530 & 2.443 \\
\hline GRU- & 0.888 & 3.220 & 1.850 & 0.939 & 3.419 & 1.920 & 1.057 & 3.889 & 2.086 \\
\hline GRU-D & 3.039 & 11.597 & 5.408 & 2.947 & 11.399 & 5.160 & 2.873 & 11.136 & 5.008 \\
\hline LSTM & 1.256 & 4.451 & 2.446 & 1.450 & 5.364 & 2.956 & 1.433 & 5.260 & 2.902 \\
\hline LSTM-I & 0.945 & 3.363 & 2.400 & 0.910 & 3.255 & 2.094 & 1.592 & 5.155 & 5.156 \\
\hline LSTM-D & 1.096 & 4.357 & 2.633 & 1.001 & 3.787 & 2.264 & 0.986 & 3.584 & 2.098 \\
\hline GMN-6 & 2.354 & 8.541 & 4.832 & 2.704 & 9.588 & 5.545 & 3.063 & 10.700 & 5.960 \\
\hline GMN-8 & 2.356 & 8.547 & 4.835 & 2.712 & 9.613 & 5.559 & 2.938 & 10.277 & 5.803 \\
\hline GMN-10 & 2.355 & 8.545 & 4.835 & 2.713 & 9.618 & 5.561 & 2.923 & 10.224 & 5.778 \\
\hline SGMN-6 & .768 & 715 & 1.922 & 0.829 & 2.940 & 2.038 & 1.355 & 4.949 & 2.983 \\
\hline SGMN-8 & .768 & 2.713 & 1.921 & 0.826 & 2.929 & 2.026 & 1.024 & 3.679 & 2.399 \\
\hline SGMN-10 & 0.768 & 2.716 & 1.921 & 0.827 & 2.934 & 2.026 & 0.973 & 3.485 & 2.283 \\
\hline
\end{tabular}

smallest ones for all missing rates. That means the GRU-I's prediction results tend to have less large errors. The test results on the INRIX-SEA dataset, as shown in Table 3, have the similar situation that SGMN models perform better in terms of the MAE and MAPE metrics and GRU-I achieves better RMSE results. As for the results tested on the METRLA dataset, shown in Table 2, the SGMN models outperform other models when the missing rates are $10 \%$ and $20 \%$. When the missing rate increases to $40 \%$, the GRU-I, LSTM-I, and LSTM-D models achieve better prediction performance in terms of all the three metrics. It should be noted that $8.11 \%$ of the values in the METR-LA dataset are originally missing. The actual missing rates of the METR-LA dataset are higher than the rates we set in the experiments. Hence, the SGMN models can achieve superior prediction results, especially 
when the missing rate is not very high.

\subsubsection{Analysis on Training Time}

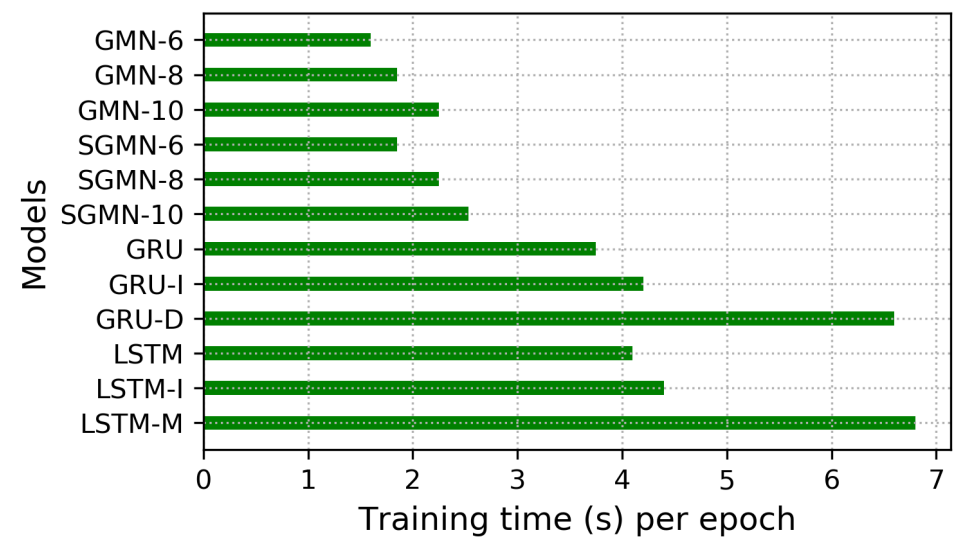

Figure 3: Training time of the compared models.

In this section, we analyze the training time of the proposed models and baseline models. Figure 3 shows the training time per epoch of the compared models tested on the PEMSBAY datasets. The training times tested on different datasets have the same patterns. Since the GMN models have less matrix product operations than the SGMN models, GMN models take slightly less time per epoch than other models. The GMN and SGMN models apparently cost less running time than the baseline models because they get rid of the recurrent structure. The training times of GMN and SGMN increase when they incorporate more historical steps. The GRU and LSTM have similar training time per epoch. Since the GRU-I and LSTM-I have an imputation operation, their training times take a little bit more. In addition, since the GRU-D and LSTM-M both take more types of data as the input, their training time is much more than GRU and LSTM.

\subsubsection{Analysis on Decay Rates of GMN and SGMN}

The proposed graph Markov process adopts the decay rate $\gamma \in(0,1)$ to represent the temporal impact of $\Delta t$ on the traffic state transition process. In previous analysis sections, the $\Delta t$ is 5 -minutes, and the decay rates of GMN models are set as 0.9 . In this section, we analyze the influence of the decay rate on the proposed models' prediction performance. The prediction performance of SGMN-10 and GMN-10 w.r.t. various decay rates are shown in Figure 4. The models are tested on the three datasets with different missing rates. Generally, the missing rate affects the prediction performance a lot that large missing rate results in large prediction errors.

The six sub-figures in Figure 4 all indicate the prediction errors (MAE) decrease along with the increase of $\gamma$. The prediction results of the SGMN-10 models tested on the three datasets have the similar curve patterns, as shown by the line-charts in Figure 4a, 4b, and 4c. When $\gamma$ is close to zero, the prediction errors are relatively large. When $\gamma$ is increasing, 


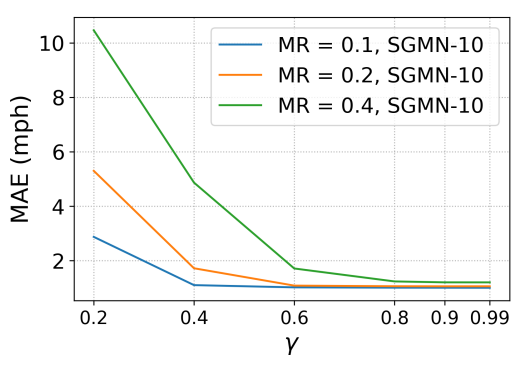

(a) SGMN-10 on PEMS-BAY

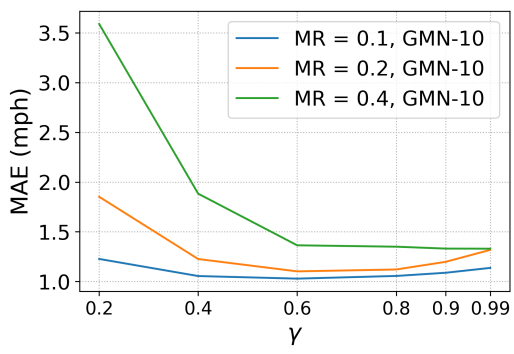

(d) GMN-10 on PEMS-BAY

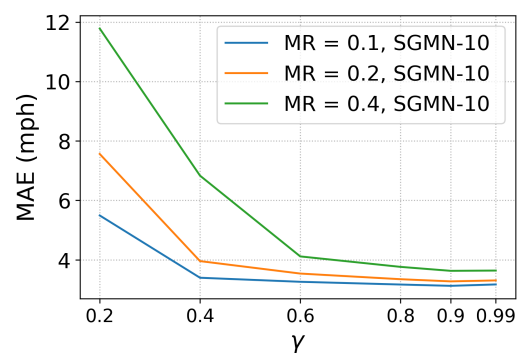

(b) SGMN-10 on METR-LA

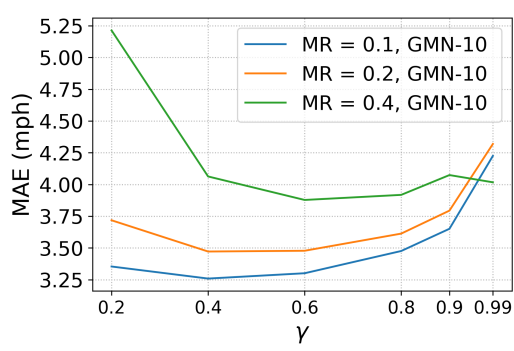

(e) GMN-10 on METR-LA

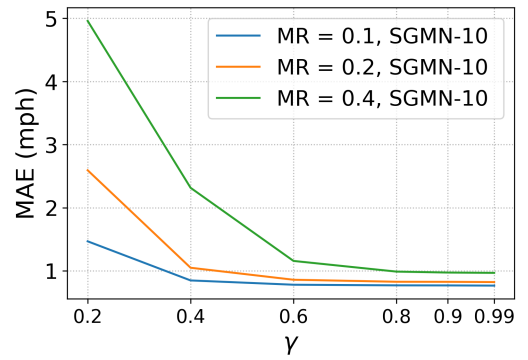

(c) SGMN-10 on INRIX-SEA

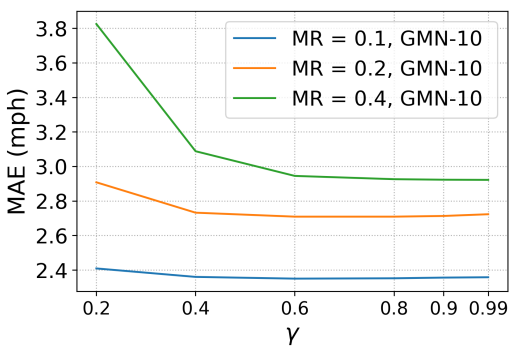

(f) GMN-10 on INRIX-SEA

Figure 4: Prediction performance metric (MAE) w.r.t. the decay rate $\gamma$. The SGMN-10 and GMN-10 are tested on the PEMS-BAY, METR-LA, and INRIX-SEA datasets with different missing rates.

the prediction errors seem to be monotonically decreasing. When $\gamma$ is close to one, the curves are almost flat and prediction errors nearly keep the same. However, as shown in Figure 4b, the MAE tested on the METR-LA dataset increases a little bit when $\gamma$ increases from 0.9 to 0.99 .

The MAEs of the GMN-10 models shown in Figure 4d, 4e, and 4f have slightly different patterns than those of SGMN-10 models. The MAE curves are not monotonically decreasing. When $\gamma$ is close to one, the prediction errors start to increase. This phenomenon is particularly obvious in the results tested on the METR-LA dataset.

In addition, it should be noted that the y-axes of those sub-figures have various ranges. When the decay rate is relatively small (close to one), the prediction capability of GMN models is better than that of SGMN models. One possible reason is that GMN models contains more weight parameters than SGMN models.

\subsubsection{Analysis on Forecasting Residuals}

Since residual is an critical indicator for evaluating whether a model is systematically correct, the residuals of predictions are analyzed in this section. The residual equals the ground truth value subtracts the predicted value, i.e. $x-\hat{x}$. Figure 5 shows the residual distributions of SGMN-10 and GMN-10 tested on the three datasets. Most of the sub-figures display that the residual distributions follow normal distributions with zero means, except for the result of GMN-10 tested on the INRIX-SEA dataset. Although the proposed models are more complex than regression models, the residuals' normal distributions indicate that the proposed models have sufficient prediction capabilities and capture enough predictive 


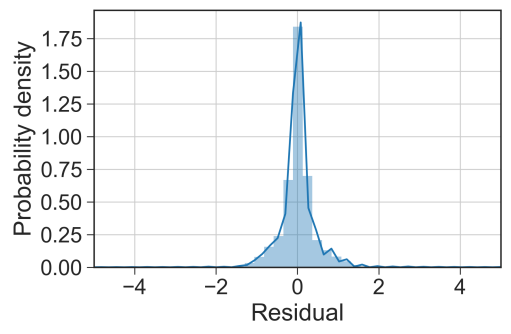

(a) SGMN-10 on PEMS-BAY

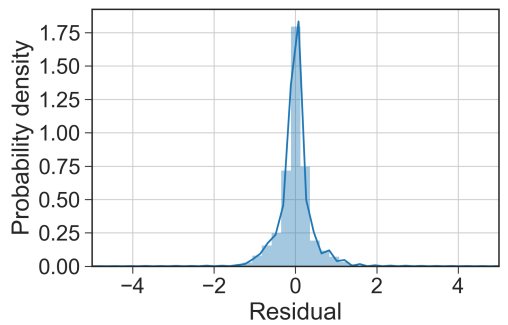

(d) GMN-10 on PEMS-BAY

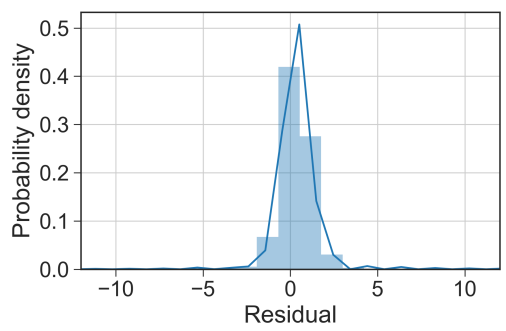

(b) SGMN-10 on METR-LA

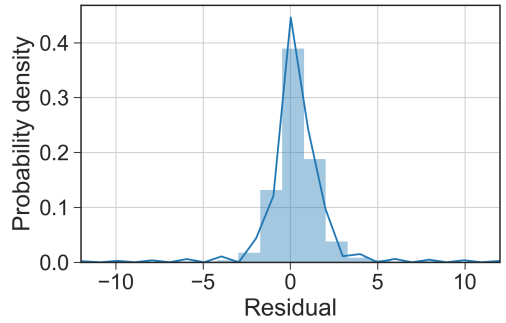

(e) GMN-10 on METR-LA

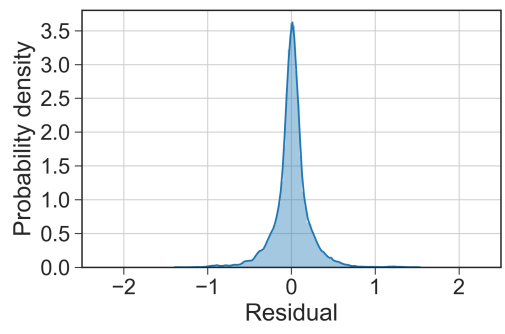

(c) SGMN-10 on INRIX-SEA

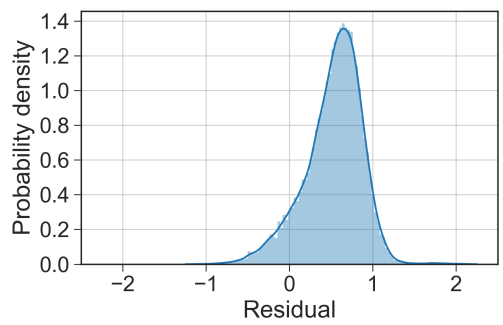

(f) GMN-10 on INRIX-SEA

Figure 5: Prediction residuals of the proposed models tested on three datasets when the missing rate is $20 \%$.

information from the input data.

The prediction performance will also be influenced by the temporal information, such as hour of day and day of the week. Normally, during peak hours, traffic states with more variations are harder to be predicted. Thus, in this section, the influence of hour of day and day of the week is measured. The residuals of SGMN-10 tested on the three datasets with respect to day of the week and hour of day are shown in Figure 6. As displayed by the box-plots in Figures 6a, 6c, 6e, the prediction residuals on each day of the week are almost the same. That means the proposed models has the capability of forecasting traffic states on each day of the week. The residuals with respect to hour of day are displayed in Figures $6 \mathrm{~b}, 6 \mathrm{~d}, 6 \mathrm{f}$. The influence of peak hours on traffic forecasting is particularly obvious on the PEMS-BAY dataset. However, the residual distributions in each hour of the day on the METR-LA dataset do not have much differences. The residual distributions on the INRIX-SEA dataset are abnormal to some extent that the the residuals are large during the afternoon and midnight. This phenomenon may be lead by the various traffic patterns of different types of roadways in different cities.

\subsubsection{Model Weight Analysis and Visualization}

In this section, the proposed model's weights are analyzed and visualized. We take the SGMN-10 and GMN-10 trained on METR-LA dataset as an example. Figure 7a shows the 207 sensor locations in the METR-LA dataset denoted by blue dots, and Figure 7b shows the top 20 most influential sensor locations in terms of the influence on forecasting traffic states of the future $(t+1)$ step from the states of the current $(t)$ step. The influence of a sensor of the $k$-th location is reflected by the sum/average of the squared element values in 


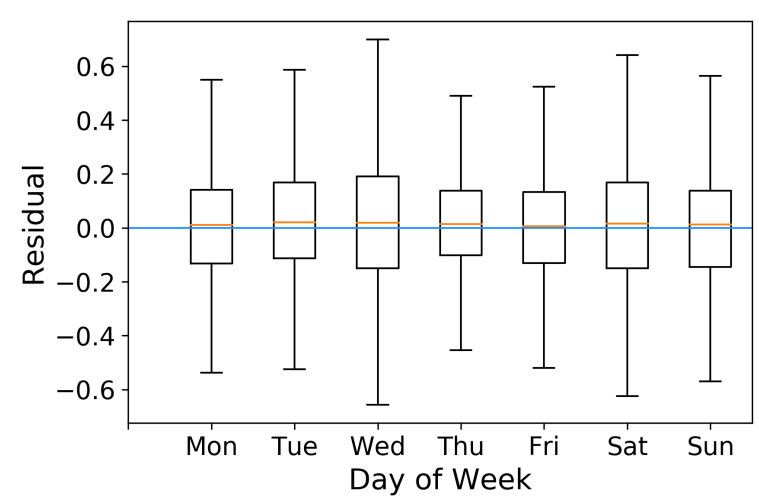

(a) PEMS-BAY

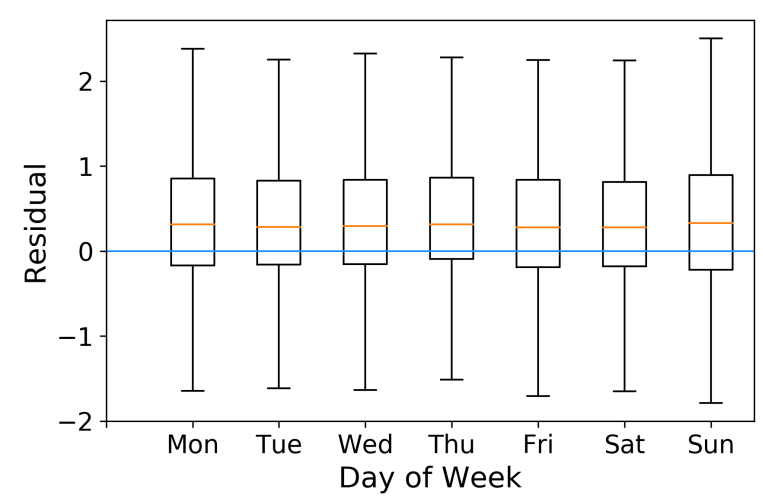

(c) METR-LA

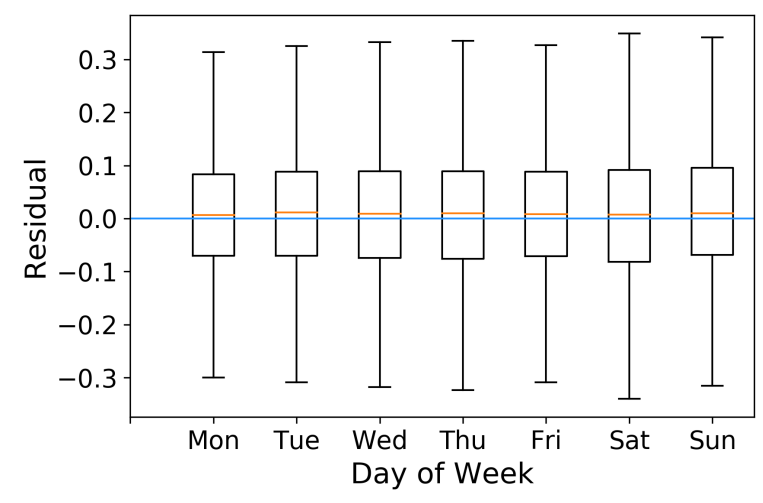

(e) INRIX-SEA

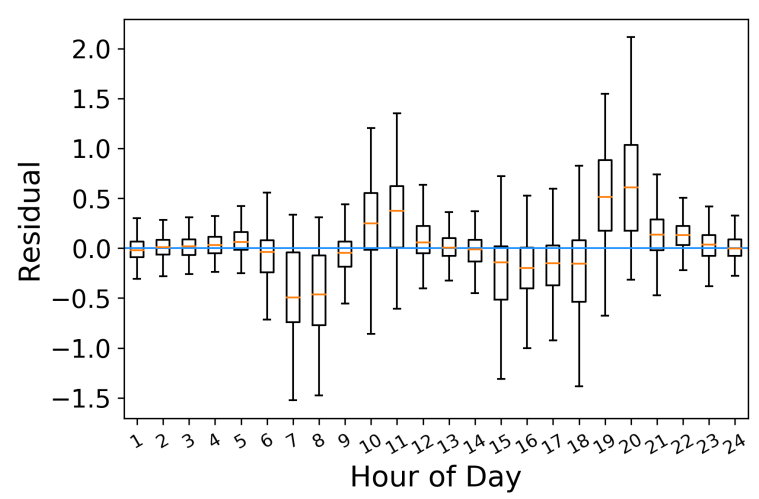

(b) PEMS-BAY

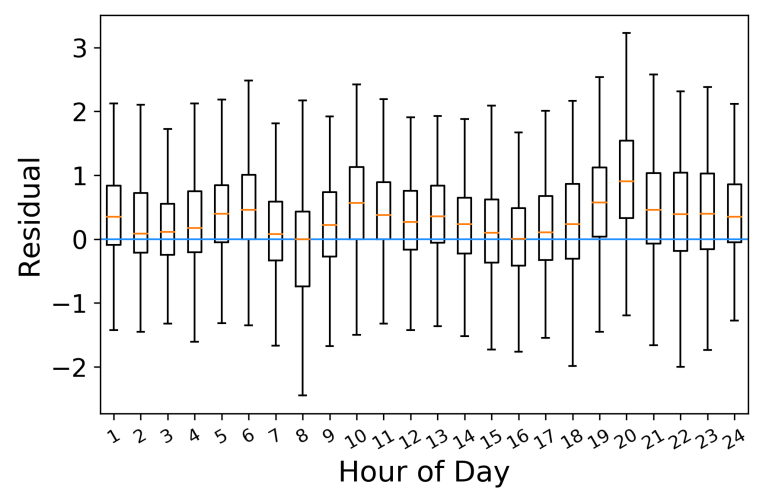

(d) METR-LA

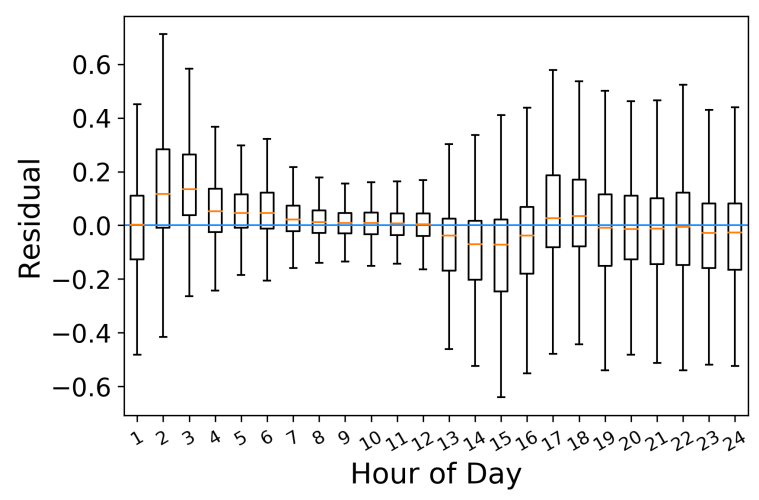

(f) INRIX-SEA

Figure 6: Prediction residuals of SGMN-10 with respect to day of the week and hour of day, tested on three datasets with the missing rate of $20 \%$.

the $k$-th row/column of the model's weight matrix at the $t$ step, i.e. the $H_{t}$ described in Equation 18. For example, the averaged squared element values of the $k$-th row of $H_{t}$ is calculated as $\frac{1}{n} \sum_{i=0}^{n-1}\left(H_{t}\right)_{k, i}^{2}$. Here, $H_{t}=\mathbf{A}^{1} \odot W_{1}$ for the GMN case, and for the SGMN case, $H_{t}=U \wedge_{1} U^{T}$. As depicted in the map in Figure 7b, the selected top 20 influential 


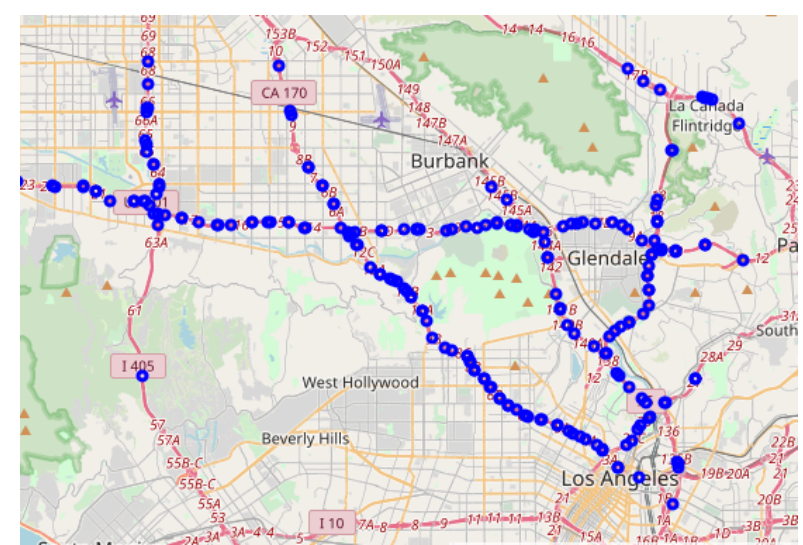

(a) All sensor locations in the METR-LA dataset

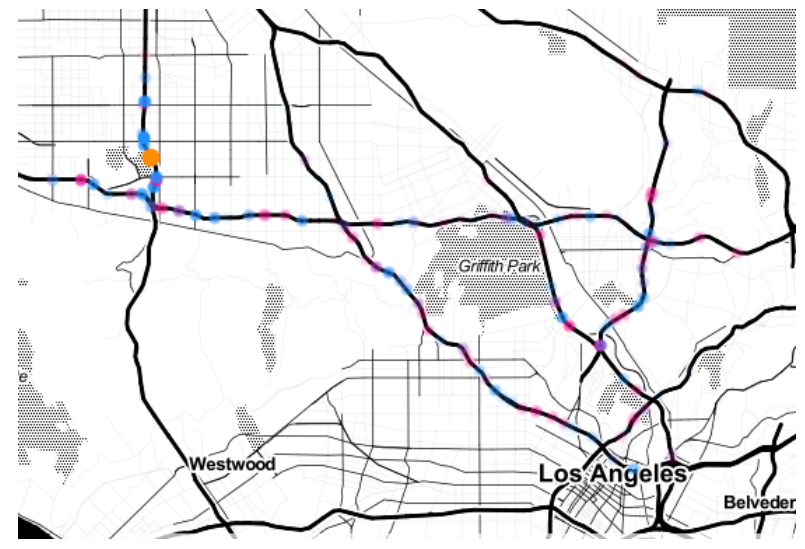

(c) Weight $\left(U \wedge_{1} U^{T}\right)$ visualization of SGMN-10 w.r.t. the $89^{\text {th }}$ sensor location, denoted by the orange dot

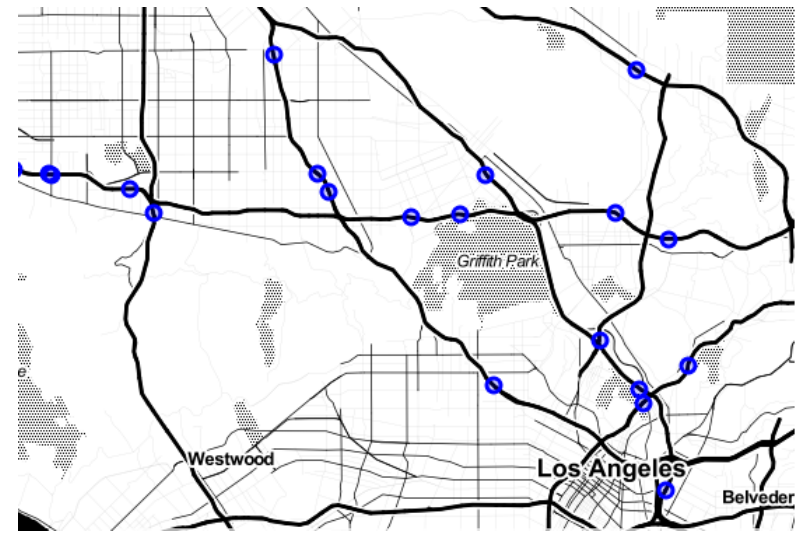

(b) Top 20 influential sensor locations

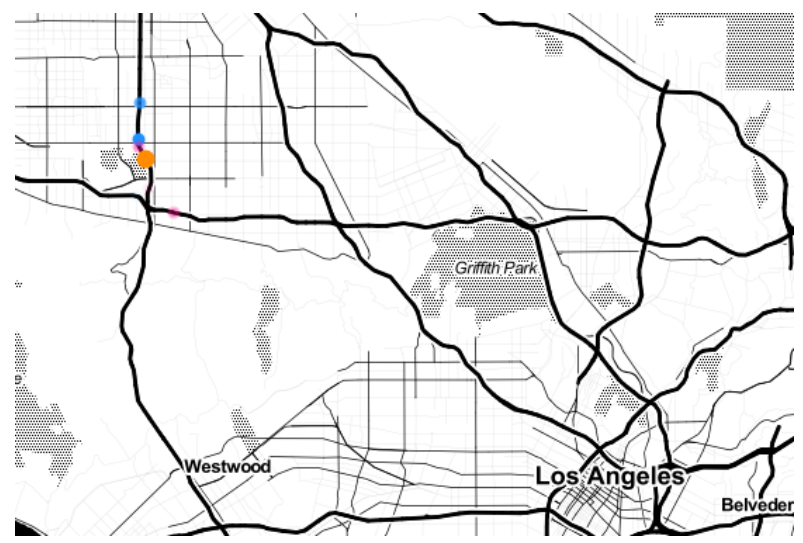

(d) Weight $\left(\mathbf{A}^{1} \odot W_{1}\right)$ visualization of GMN-10 w.r.t. the $89^{\text {th }}$ sensor location, denoted by the orange dot

Figure 7: Visualization of sensor locations and models weights. The top 20 influential sensor locations in (b) are ones with the top 20 largest row-wise averaged squared element values of the weight matrix $H_{1}=U \Lambda_{1} U^{T}$ in SGMN-10, which is introduced in Section 5.8.5. The blue and pink dots in (c) and (d) represent positive and negative weight values, respectively. The darker the color is, the larger the absolute value of the weight is.

sensor locations are mostly distributed near intersection areas, which has great potential to affect nearby traffic states. Figure 7c and Figure 7d display the influence of the 89-th sensor location on its neighboring sensor locations. This sensor location with the sensor ID 767351 is represented by an orange dot on the maps. The influence is reflected by the element values of the model's weight matrix at the 89-th row/column. The positive and negative weight values of other sensor locations are demonstrated by blue and pink colors, respectively. The darker the color is, the larger the absolute value of the weight element is. The difference between these two figures is that the illustrated neighboring locations in Figure $7 \mathrm{~d}$ are confined within a small one-hop neighboring area by the weight matrix of GMN-10, i.e. the $\mathbf{A}^{1} \odot W_{1}$. However, as shown by the two figures, the surrounding sensor 
locations with respect to the 89-th sensor location (the orange dot) are obviously darker, which means the traffic state of a location is influenced more by the states of its neighbors. Thus, by quantitatively analyzing or visualizing the weight matrices of the proposed models, the influence of nodes/locations in a traffic network on their neighbors can be measured.

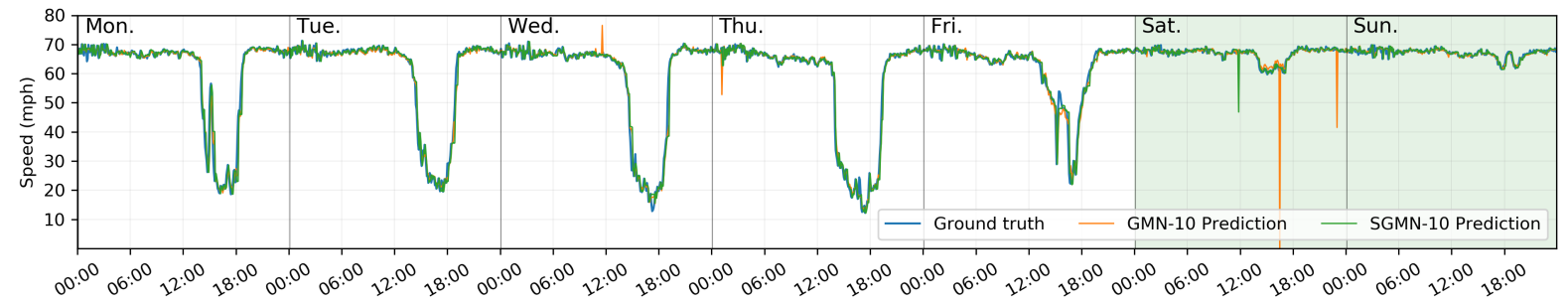

(a) Results tested on the PEMS-BAY dataset from Jan. 30, 2017 to Feb. 5, 2017. Sensor ID = 400097

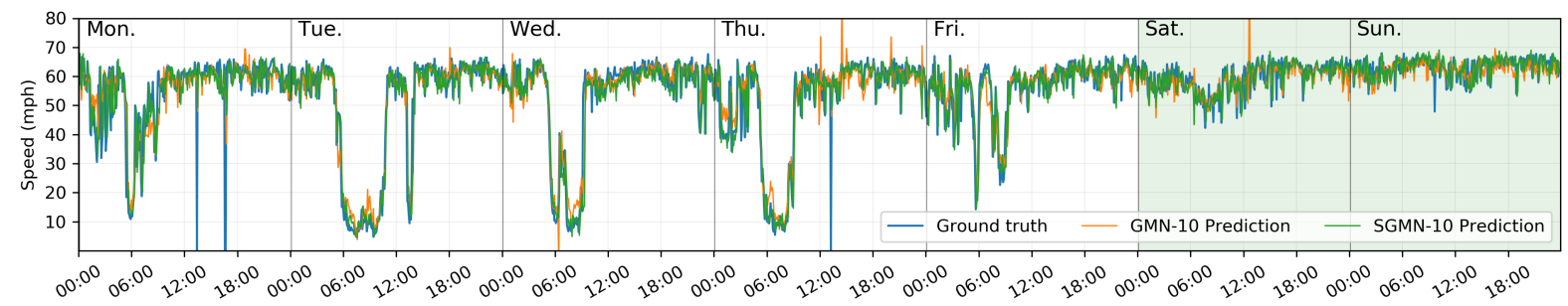

(b) Results tested on the METR-LA dataset from Mar. 12, 2012 to Mar. 18, 2012. Sensor ID = 765273

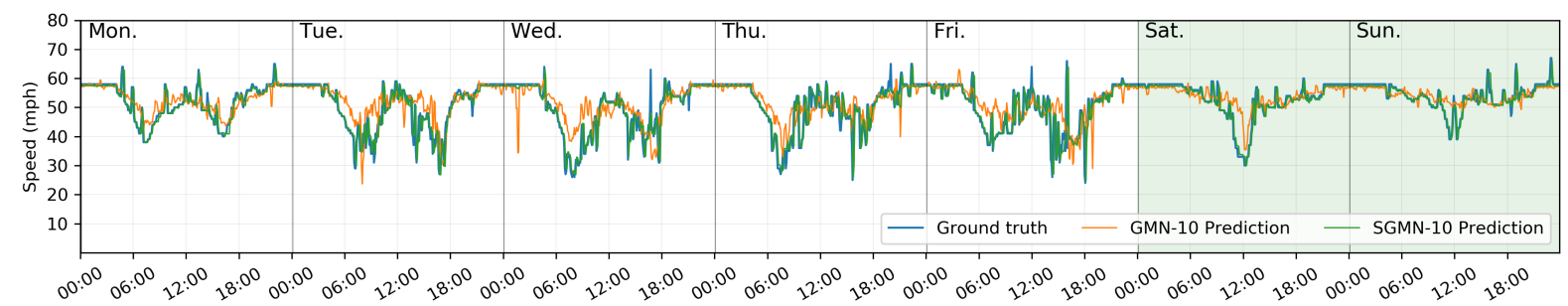

(c) Results tested on the INRIX-SEA dataset from Jan. 1, 2012 to Jan. 7, 2012. link ID = $114+04549$

Figure 8: Comparison of the ground truth and the speed predicted by GMN-10 and SGMN-10 tested on three datasets with the missing rate of $20 \%$ under the random missing scenario. The white and green regions in these figures demonstrate weekdays and weekends, respectively.

\subsubsection{Traffic Forecasting Result Visualization}

The locations covered by those datasets actually have various traffic patterns. In this section, to demonstrate the proposed model's prediction performance, we select several sensor locations/links from the three datasets and visualize the ground truth and predicted speed values. The three sub-figures in Figure 8 display the ground truth and speed values predicted by the GMN-10 and SGMN-10. The missing rates of the tested datasets are all set as 20\%. Both GMN-10 and SGMN-10 work well on the PEMS-BAY dataset. Since 
the METR-LA dataset originally has missing values, there are some blue spikes reaching the bottom of Figure 8b demonstrating the original missing values. The prediction performance of SGMN-10 on the INRIX-SEA dataset is better than that of GMN-10. Overall, the proposed models have the capability of forecasting traffic states with missing values.

\section{Conclusion}

In this study, we propose the GMN, which is a new neural network architecture for spatial-temporal data forecasting. We introduce two properties of the traffic state transition process and define a graph Markov process. Unlike other existing recurrent neural network (RNN)-based models dealing with traffic data as multivariate time series, the GMN handling the traffic state transition process as a graph Markov process. The proposed GMN can incorporate the spatial relationship between neighboring links and the links' temporal dependencies between different time steps. By incorporating the spectral graph convolution operation, we also propose a spectral graph Markov network (SGMN). The experimental results tested on a real-world dataset shows show that the proposed GMN and SGMN achieves superior prediction performance. Further, the proposed models' parameters, weights, and prediction residuals are discussed and visualized.

The future work will focus on enhancing the theoretical basis of the proposed graph Markov process. We will attempt to build a connection between the graph Markov process with the Markov random field to analyze the hidden factors influencing traffic states. In addition, we will conduct more experiments on multiple public accessible datasets.

\section{Acknowledgments}

This work was supported by the Connected Cities with Smart Transportation (C2SMART) Tier 1 University Transportation Center with the USDOT Award No.: 69A3551747124. Thanks to Washington State Department of Transportation (WSDOT) for providing the research datasets. Thanks to Xinyu Chen for sharing the academic-drawing code on GitHub. Also, the authors would like to thank Ruimin Ke and Shuyi Yin for helpful discussions and comments.

\section{Reference}

\section{References}

[1] I. Laña, I. I. Olabarrieta, M. Vélez, J. Del Ser, On the imputation of missing data for road traffic forecasting: New insights and novel techniques, Transportation research part C: emerging technologies 90 (2018) 18-33.

[2] E. I. Vlahogianni, M. G. Karlaftis, J. C. Golias, Short-term traffic forecasting: Where we are and where were going, Transportation Research Part C: Emerging Technologies 43 (2014) 3-19.

[3] X. Chen, Z. He, L. Sun, A bayesian tensor decomposition approach for spatiotemporal traffic data imputation, Transportation research part C: emerging technologies 98 (2019) 73-84.

[4] J. Yoon, J. Jordon, M. Schaar, Gain: Missing data imputation using generative adversarial nets, in: International Conference on Machine Learning, 2018, pp. 5675-5684. 
[5] Y. Zhang, Y. Zhang, A comparative study of three multivariate short-term freeway traffic flow forecasting methods with missing data, Journal of Intelligent Transportation Systems 20 (3) (2016) 205-218.

[6] S. Lee, D. B. Fambro, Application of subset autoregressive integrated moving average model for shortterm freeway traffic volume forecasting, Transportation Research Record 1678 (1) (1999) 179-188.

[7] Y. Duan, Y. Lv, Y.-L. Liu, F.-Y. Wang, An efficient realization of deep learning for traffic data imputation, Transportation research part C: emerging technologies 72 (2016) 168-181.

[8] Y. Tian, K. Zhang, J. Li, X. Lin, B. Yang, Lstm-based traffic flow prediction with missing data, Neurocomputing 318 (2018) 297-305.

[9] B. M. Williams, Multivariate vehicular traffic flow prediction: evaluation of arimax modeling, Transportation Research Record 1776 (1) (2001) 194-200.

[10] I. Okutani, Y. J. Stephanedes, Dynamic prediction of traffic volume through kalman filtering theory, Transportation Research Part B: Methodological 18 (1) (1984) 1-11.

[11] B. Ghosh, B. Basu, M. O'Mahony, Multivariate short-term traffic flow forecasting using time-series analysis, IEEE transactions on intelligent transportation systems 10 (2) (2009) 246.

[12] B. L. Smith, B. M. Williams, R. K. Oswald, Comparison of parametric and nonparametric models for traffic flow forecasting, Transportation Research Part C: Emerging Technologies 10 (4) (2002) 303-321.

[13] C.-H. Wu, J.-M. Ho, D.-T. Lee, Travel-time prediction with support vector regression, IEEE transactions on intelligent transportation systems 5 (4) (2004) 276-281.

[14] S. Sun, C. Zhang, G. Yu, A bayesian network approach to traffic flow forecasting, IEEE Transactions on intelligent transportation systems 7 (1) (2006) 124-132.

[15] X. Ma, Z. Tao, Y. Wang, H. Yu, Y. Wang, Long short-term memory neural network for traffic speed prediction using remote microwave sensor data, Transportation Research Part C: Emerging Technologies 54 (2015) 187-197.

[16] Z. Cui, R. Ke, Y. Wang, et al., Deep stacked bidirectional and unidirectional lstm recurrent neural network for network-wide traffic speed prediction, in: 6th International Workshop on Urban Computing (UrbComp 2017), 2016.

[17] X. Ma, Z. Dai, Z. He, J. Ma, Y. Wang, Y. Wang, Learning traffic as images: a deep convolutional neural network for large-scale transportation network speed prediction, Sensors 17 (4) (2017) 818.

[18] Y. Liang, Z. Cui, Y. Tian, H. Chen, Y. Wang, A deep generative adversarial architecture for networkwide spatial-temporal traffic-state estimation, Transportation Research Record 2672 (45) (2018) 87105.

[19] H. Yang, C. Liu, C. Gottsacker, X. Ban, C. Zhang, Y. Wang, Cell-speed prediction neural network (cpnn): A deep learning approach for trip-based speed prediction, Tech. rep. (2019).

[20] H. Yu, Z. Wu, S. Wang, Y. Wang, X. Ma, Spatiotemporal recurrent convolutional networks for traffic prediction in transportation networks, Sensors 17 (7) (2017) 1501.

[21] X. Ma, Y. Li, Z. Cui, Y. Wang, Forecasting transportation network speed using deep capsule networks with nested lstm models, arXiv preprint arXiv:1811.04745.

[22] B. Yu, H. Yin, Z. Zhu, Spatio-temporal graph convolutional networks: A deep learning framework for traffic forecasting, arXiv preprint arXiv:1709.04875.

[23] Z. Cui, K. Henrickson, R. Ke, Y. Wang, Traffic graph convolutional recurrent neural network: A deep learning framework for network-scale traffic learning and forecasting, arXiv preprint arXiv:1802.07007.

[24] Y. Li, R. Yu, C. Shahabi, Y. Liu, Diffusion convolutional recurrent neural network: Data-driven traffic forecasting, arXiv preprint arXiv:1707.01926.

[25] L. Li, X. Su, Y. Zhang, J. Hu, Z. Li, Traffic prediction, data compression, abnormal data detection and missing data imputation: An integrated study based on the decomposition of traffic time series, in: 17th International IEEE Conference on Intelligent Transportation Systems (ITSC), IEEE, 2014, pp. $282-289$.

[26] B. Ran, H. Tan, Y. Wu, P. J. Jin, Tensor based missing traffic data completion with spatial-temporal correlation, Physica A: Statistical Mechanics and its Applications 446 (2016) 54-63.

[27] X. Chen, Z. He, L. Sun, A Bayesian tensor decomposition approach for spatiotemporal traffic data imputation, Transportation Research Part C: Emerging Technologies 98 (2019) 73-84. doi:10.1016/ 
j.trc.2018.11.003.

URL https://www.sciencedirect.com/science/article/pii/S0968090X1830799X

[28] X. Chen, Z. He, J. Wang, Spatial-temporal traffic speed patterns discovery and incomplete data recovery via svd-combined tensor decomposition, Transportation research part C: emerging technologies 86 (2018) 59-77.

[29] J. Tang, G. Zhang, Y. Wang, H. Wang, F. Liu, A hybrid approach to integrate fuzzy c-means based imputation method with genetic algorithm for missing traffic volume data estimation, Transportation Research Part C: Emerging Technologies 51 (2015) 29-40.

[30] W. C. Ku, G. R. Jagadeesh, A. Prakash, T. Srikanthan, A clustering-based approach for data-driven imputation of missing traffic data, in: 2016 IEEE Forum on Integrated and Sustainable Transportation Systems (FISTS), IEEE, 2016, pp. 1-6.

[31] Z. Che, S. Purushotham, K. Cho, D. Sontag, Y. Liu, Recurrent neural networks for multivariate time series with missing values, Scientific reports 8 (1) (2018) 6085.

[32] M. Defferrard, X. Bresson, P. Vandergheynst, Convolutional neural networks on graphs with fast localized spectral filtering, in: Advances in neural information processing systems, 2016, pp. 3844-3852.

[33] H. Rue, L. Held, Gaussian Markov random fields: theory and applications, Chapman and Hall/CRC, 2005.

[34] X. Wang, P. Van Mieghem, Orthogonal eigenvector matrix of the laplacian, in: 2015 11th International Conference on Signal-Image Technology \& Internet-Based Systems (SITIS), IEEE, 2015, pp. 358-365.

[35] Y. Li, R. Yu, C. Shahabi, Y. Liu, Diffusion convolutional recurrent neural network: Data-driven traffic forecasting, in: International Conference on Learning Representations (ICLR '18), 2018.

[36] H. Jagadish, J. Gehrke, A. Labrinidis, Y. Papakonstantinou, J. M. Patel, R. Ramakrishnan, C. Shahabi, Big data and its technical challenges, Communications of the ACM 57 (7) (2014) 86-94.

[37] K. Cho, B. van Merrienboer, C. Gulcehre, D. Bahdanau, F. Bougares, H. Schwenk, Y. Bengio, Learning phrase representations using rnn encoder-decoder for statistical machine translation, in: Proceedings of the 2014 Conference on Empirical Methods in Natural Language Processing (EMNLP), 2014, pp. 1724-1734.

[38] S. Hochreiter, J. Schmidhuber, Long short-term memory, Neural computation 9 (8) (1997) 1735-1780.

[39] D. P. Kingma, J. Ba, Adam: A method for stochastic optimization, arXiv preprint arXiv:1412.6980. 